\title{
A ADESÃO AO PROTOCOLO DE MONITORAMENTO DOSTRABALHADORES DE SAÚDE APÓS EXPOSIÇÃO A FLUIDOS BIOLÓGICOS: UMA PROBLEMÁTICA VIVENCIADA EM UM AMBULATÓRIO DE SAÚDE DO TRABALHADOR NO PARANÁ*
}

[Health workers' compliance to the monitoring protocol after exposure to biological fluids: a problem experienced in an outpatient clinic at the worker's hospital in Paraná]

[Adhesión al protocolo de monitorización de los trabajadores de salud después de exposición a fluidos biológicos: una problemática vivida en un ambulatorio de salud del trabajador en Paraná]

Leila M aria M ansano Sarquis** Vanda Elisa A ndres Felli***

Fernanda M oura D 'A Imeida M iranda**** Hermman Valentin Guimarães*****

Giordano Pedro de Oliveira******

RESUMO: Pesquisa epidemiológica, estudo de coorte, com abordagem quantitativa, com os objetivos: caracterizar 0 perfil dos trabal hadores acidentados com fluidos biológicos e analisar a adesão quanto ao retorno ao ambulatório para consulta após a exposição ocupacional. A instituição campo de estudo foi o A mbulatório do Trabal hador pertencente ao Hospital do Trabalhador da U niversidade Federal do Paraná, situado em Curitiba, Paraná. Foram analisados 491 prontuários no período de abril a dezembro de 2004. Os dados foram coletados mediante Ficha de A companhamento de Acidente com Material Biológico e do Boletim de Emergência no Pronto Socorro desta instituição. Os dados mostram que a maioria dos sujeitos pertence ao sexo feminino $(80,4 \%)$, com média de idade de 21 a 25 anos $(25,8 \%)$. A categoria auxiliar de enfermagem foi a que mais

\footnotetext{
*Pesquisa desenvolvida na U nidade Saúde do Trabalhador na cidade de Curitiba, Paraná.

**Enfermeira, Profa. do Departamento de Enfermagem da U niversidade Federal do Paraná - UFPR, membro do GEM SA (Grupo multiprofissional de saúde do adulto), aluna doutoranda do Programa de Pós-graduação pela Escola de Enfermagem - Universidade de São Paulo, B rasil, bolsista CN Pq. Endereço: Rua Padre Camargo 280, 8 andar, CEP: 80 060-240. Curitiba, PR, B rasil (41) 3360-7252 e mail: m.sarquis@brturbo.com.br

***Enfermeira, Profa. Dra Orientadora A ssociada do Departamento de Orientação Profissional da Escola de Enfermagem da U niversidade de São Paulo.

****Enfermeira da Unidade Saúde do Trabal hador do Hospital do Trabalhador.

*****M édico especial ista em M edicina do Trabalho, coordenador da Unidade Saúde do Trabalhador, diretor técnico do Hospital do Trabalhador.

******A cadêmico do Curso de Graduação de Enfermagem da UFPR, bolsista voluntário.
}

entrou em contato com fluídos biológicos (46\%), os funcionários que executam atividade de limpeza em unidades de saúde também se acidentam em uma amostra significativa (12\%). Dos 491 prontuários registrados que deram entrada no Serviço de Emergência por exposição ocupacional, apenas $175(35,6 \%)$ retornaram para a segunda consulta ambulatorial após 30 dias do acidente. Para a consulta previamente agendada após 90 dias do acidente apenas $103(21 \%)$. Os registros mostraram que apenas 50 trabal hadores $(10,2 \%)$ fizeram o monitoramento e acompanhamento completo neste ambulatório. Em relação ao tipo de acidente encontramos um percentual significativo de $88,6 \%$ dos trabalhadores que sofreram acidente com instrumento perfurocortante. A penas uma pequena amostra de $11,4 \%$ entrou em contato com os fluidos biológicos através de respingos em mucosas oculares. A creditamos que este estudo pode subsidiar a reflexão dos administradores e dos trabalhadores de saúde para o desenvolvimento de estratégias que possibilitem minimizar a exposição aos riscos a que estes trabalhadores estão expostos, e maximizar os recursos institucionais para a realização do monitoramento completo quando expostos a fluidos biológicos.

PALAVRAS-CHAVE: A cidentes de trabalho; Saúde ocupacional; Exposição ocupacional.

\section{IINTRODUÇÃO}

$\mathrm{N}$ os dias atuais, os acidentes de trabal ho que acometem os trabalhadores de enfermagem são representados pel os ferimentos pérfurocortantes, sendo um 
grave problema nas instituições de saúde, tanto pela freqüência com que ocorrem, como pela grave repercussão que representam na saúde desses trabalhadores. 0 conhecimento a respeito desses acidentes, sua prevenção e controle constituem um desafio a ser enfrentado, tanto por essas instituições, como pelos diferentes níveis dos órgãos municipais, estaduais e federais incumbidos dessa responsabilidade.

Com o processo da implantação do Sistema Ú nico de Saúde no Brasil e no Paraná e com o avanço da municipalização, às Secretarias Estaduais de Saúde coube um novo papel, de apoio e cooperação técnica e investimentos nos municípios, reduzindo a taxa de ações e serviços que foi sendo assumida pelos municípios e consórcios segundo a sua capacidade. Com isto, a Secretaria de Saúde do Estado apóia, investiga e desenvolve ações complementares e mecanismos capazes de organizar e sustentar o funcionamento do Sistema Estadual de Saúde ${ }^{(1)}$. A partir destes dados e considerando a escassez de estudos mais aprofundados a respeito dos acidentes de trabal ho, justificou-se proceder o estudo com trabal hadores de saúde no A mbulatório de Saúde do Trabalhador, em Curitiba, Paraná.

Na Secretaria de Saúde do Estado do Paraná, a área de Saúde do Trabal hador começou a se estruturar no início da década de noventa com a criação de um grupo de Coordenação em Saúde do Trabalhador, composto por técnicos da Vigilância Epidemiológica e daA ssistência. N este período foram promovidos alguns treinamentos para os técnicos das Regionais de saúde de al guns municípios ${ }^{(1)}$.

\subsection{OBJETIVOS}

Caracterizar o perfil dos trabalhadores acidentados com material biológico e anal isar a adesão dos trabalhadores quanto ao retorno ao ambulatório para consulta após a exposição ocupacional.

\section{DESENVOLVIMENTO}

No B rasil, os estudos que enfocam a relação saúdetrabalho nas instituições hospitalares, começaram a ser realizados na década de 70 do século passado e foram incrementados a partir da década de 80 . A pesar de ter havido um incremento nestas publicações na última década, estas ainda não foram suficientes para caracterizar quantiqualitativamente os problemas específicos da relação trabalho-saúde (2). Estes estudos demonstram que a saúde do trabal hador está comprometi da e este comprometimento, em parte, pode ser detectado através da el evada incidência de acidentes de trabalho e doenças profissionais (3).

A lguns dos estudos, desenvolvidos em nosso país, na última década, permitem situar e contextualizar a problemática de saúde vivenciada pelos trabal hadores de instituições de saúde $(4,5,3,6,7,8,9,2,10,11,12,13,14,15,16,17,18,19,20,21$,
${ }^{22,23)}$, sendo que, os autores abordam a saúde do trabal hador e a sua relação com o trabalho.

Com a preocupante questão relacionada aos riscos ocupacionais associados à exposição do trabalhador, algumas pesquisas foram desenvolvidas com o objetivo de procurar explicar o comportamento de profissionais de enfermagem em sua prática. Baseada no M odelo de Crenças em Saúde (Health Belief Model), B revidelli (11) buscou quantificar as crenças individuais que poderiam estar influenciando na prática de reencapar agulhas e encontrou que as percepções de barreiras físicas, cognitivas e psicológicas foram as crenças mais importantes para explorar o comportamento destes profissionais. Estes resultados encontrados fornecem embasamento para estratégias de intervenção destinadas a provocar mudanças de comportamento. Um outro dado encontrado, é que os trabal hadores formados antes de 1988, ano da di vul gação das Precauções Universais, são os mais resistentes à adesão das recomendações. Esta teoria estudada por Brevidelli (11), se contrapõe aos estudos que enfocam a geração de acidentes de trabalho e a correlação existente no processo saúde-doença sob a luz da determinação social na relação trabalho-saúde já estudados por vários pesquisadores $(2,13,8)$.

Essa preocupação não é recente, pois já em 1981 representantes da Organização M undial de Saúde (OM S), reunidos em $\mathrm{H}$ aya, reconheceram não dispor de estatísticas nacionais e internacionais sobre acidentes e lesões que afetam os profissionais de saúde e dentre eles os trabalhadores de enfermagem ${ }^{(24)}$. Com isso, verificamos que, apesar dos esforços despendidos em quase duas décadas, poucas mudanças ocorreram nesse sentido.

Estes acidentes de trabalho são muitas vezes subnotificados, de acordo com estudos realizados $(10,11,13,25,26)$. Às vezes 0 empregador não está ciente da ocorrência do acidente, porém lembramos que de qualquer forma, a lei indica a responsabilidade do empregador na saúde do trabalhador de forma soberana sobre qualquer número ou locus.

0 interesse pela questão do acidente de trabal ho com instrumentos pérfurocortantes tornou-se mais evidente quando a A IDS (Síndrome da ImunodeficiênciaA dquirida) se expandiu, tornando-se uma pandemia. Alguns profissionais de saúde foram contaminados pelo vírus da AIDS, o HIV (vírus da imunodeficiência humana), no trabalho. Estes casos foram confirmados e descritos pelo Centers for Disease Control - (CDC), somando 57casos de soroconversão após exposição ocupacional, e 138 casos de possíveis soroconversões por exposição ocupacional $(27,28)$.

\section{METODOLOGIA}

Pesquisa epidemiológica, estudo de coorte, com abordagem quantitativa. A instituição campo de estudo foi - A mbulatório do Trabalhador pertencente ao Hospital do Trabalhador da Universidade Federal do Paraná, situado 
na cidade de Curitiba, Paraná. Este estudo utilizou de recursos exploratório e descritivo caracterizando os trabal hadores acidentados com material biológico.

Pesquisa quantitativa, do tipo descritiva documental. A amostragem foi intencional ou por seleção racional. 0 pesquisador, segundo Polit e Hungler (29:148) pode decidir selecionar intencionalmente os seus sujeitos tidos como característicos da população em questão, ou particul armente, conhecedores das questões que estão sendo usadas.

Foram analisados 491 prontuários no período de abril a dezembro de 2004. Os dados foram coletados através da Ficha de A companhamento de A cidente com M aterial Biológico e através do Boletim de Emergência no Pronto Socorro desta instituição, tabulados estatisticamente e discutidos em planilhas Excel.

Vamos destacar alguns dados com a finalidade de apresentar a instituição onde foi desenvolvido o estudo. A o longo de mais de 50 anos, o Hospital do Trabal hador vem tril hando uma história de assistência à saúde com inegáveis benefícios à comunidade de Curitiba e do Paraná. A instituição começou como um centro de tratamento médico de abrangência localizada e foi se tornando referência no atendimento de diversas especialidades médicas, exigindo cada vez mais infra-estrutura, capacitação profissional e mel hor desenvolvimento gerencial.

A pós 14 anos, o Hospital Geral do Portão passou por reforma, sendo então, re-inaugurado em 1994 com 0 nome de Hospital Geral M auro Senna Goulart. No ano seguinte em 1995 ocorreu a inauguração da $M$ aternidade Dr. L uiz F. Cajado de Oliveira B raga. Somente no ano de 1997, transformou-se em Hospital do Trabalhador. Neste mesmo ano, foi assinado um convênio entre a Secretaria Estadual de Saúde, a Prefeitura M unicipal de Curitiba, a Universidade Federal do Paraná/Setor Ciências da Saúde, e a Fundação para o Desenvolvimento da Ciência, da Tecnologia e da Cultura (FUNPAR), transformando o Hospital M auro Senna Goulart em Hospital do Trabal hador voltado ao atendimento dessa clientela e do públ ico em geral e à capacitação de alunos, residentes e pessoal técnico administrativo. Sua missão foi redefinida tornando-se hospital especializado, no atendimento ao trauma e nas doenças relacionadas ao trabal ho.

A tualmente, a Saúde do Trabalhador está contemplada no Sistema Ú nico de Saúde (SUS), a partir da Constituição de 1988, em seu artigo 200, inciso II, atribuindo competência ao SUS de executar ações de prevenção e reabilitação da Saúde ao Trabal hador. Em 1996, foi criado o CEM AST (Centro M etropolitano de A poio à Saúde do Trabalhador), de abrangência macro-regional, como referência na Saúde do Trabalhador, visando o estabelecimento de nexo causal das doenças profissionais. Foram criados ainda o Comitê Estadual de Investigação de Óbitos e A mputações Relacionados ao Trabalho e três Grupos Interinstitucionais de Estudos em Saúde do
Trabal hador (Lesões por Esforço Repetitivo, A mputação e Perda A uditiva induzida por Ruído). No ano seguinte, em 1999, foi criado o Departamento de Saúde no Trabalho estruturado no Centro de Saúde A mbiental desta Secretaria que tem por objetivo coordenar a política de Saúde do Trabalhador no SUS e elaborar um Programa de Saúde do Trabal hador para os servidores do Instituto de Saúde do Paraná (ISEP) (1).

Em 1999, ainda, o Hospital do Trabalhador implantou o Centro de Estudos, Pesquisa e D esenvolvimento Humano, que assumiu a administração de estágios curriculares e extracurriculares de alunos, proveniente de diversas instituições de ensino. Logo, o Hospital do Trabal hador foi reconhecido pelo M inistério da Educação e da Cultura e pelo M inistério da Saúde como "Hospital A uxiliar de Ensino", cooperando com a formação de profissionais de saúde e, por essa razão, passou a receber recursos adicionados para o seu custeio. Dessa forma, 0 convênio teve impacto positivo também nas atividades de ensino e pesquisa, propiciando ao Hospital do Trabalhador estar em permanente evolução, em sintonia com os novos conhecimentos científicos e com as novas técnicas de cada especialidade.

A participação da Fundação da U niversidade Federal do Paraná para O Desenvolvimento da Ciência, da Tecnologia e da Cultura (FUNPAR) nesse convênio proporciona autonomia e flexibilidade administrativa para vinculação de pessoas, aquisição de insumos e equipamentos, realização de contratos e ações de manutenção em geral. Credenciada no SU S como prestadora de serviços, pelo hospital, a FUNPA R recebe e administra os recursos desse faturamento e também viabiliza o uso de recursos financeiros de outras fontes de financiamento, para 0 custeio de unidade.

Com recursos administrados pela FUNPAR, oriundos do faturamento do SUS, do Fundo de Incentivo ao Desenvolvimento de Ensino e Pesquisa em Saúde (FIDEPS) e dos subsídios repassados pela Secretaria de Estado da Saúde do Paraná, foi possível a realização de melhorias na estrutura física e aquisição de equipamentos para o Hospital.

Em 2000, realizou-se a Conferência Estadual de Saúde do Trabalhador. A Secretaria firmou convênio com o M inistério da Saúde em dezembro de 2000 para a Implantação em Unidades de Saúde de Serviço para Tratamento dos A gravos relativos a Saúde do Trabal hador, que envolve a realização de treinamentos e a aquisição de equipamentos para a implantação de 05 Centros de Referência em pólos macro-regionais ${ }^{(1)}$.

\section{RESULTADOS}

D os 491 prontuários registrados que adentraram no Serviço de Emergência por exposição ocupacional, a maior exposição está entre as mul heres (80.4\%). A média de idade 
estava entre 21-25 anos (25.8\%), e a grande exposição está as funcionárias da limpeza nas unidades hospitalares (12\%), entre os auxiliares de enfermagem (46\%), e posteriormente conforme verificamos na tabela 1:

Tabela 1 - A presentação esquemática da exposição a fluidos biológicos em relação ao sexo. Curitiba, 2004

\begin{tabular}{llllllc}
\hline Mês & \multicolumn{2}{l}{ Sexo Feminino } & \multicolumn{2}{l}{ Sexo Masculino } & \multicolumn{2}{l}{ Total } \\
\hline \multirow{2}{*}{ Abril } & $\mathrm{N}^{\circ}$ & $\%$ & $\mathrm{~N}^{\circ}$ & $\%$ & $\mathrm{~N}^{\circ}$ & $\%$ \\
Maio & 56 & 75,0 & 17 & 25,0 & 69 & 100,0 \\
Junho & 33 & 83,3 & 7 & 16,7 & 41 & 100,0 \\
Julho & 33 & 80,0 & 10 & 20,0 & 52 & 100,0 \\
Agosto & 60 & 90,9 & 7 & 9,1 & 68 & 100,0 \\
Setembro & 34 & 72,0 & 13 & 28,0 & 47 & 100,0 \\
Outubro & 66 & 95,0 & 4 & 5,0 & 70 & 100,0 \\
Novembro & 32 & 70,4 & 14 & 29,6 & 47 & 100,0 \\
Dezembro & 49 & 78,1 & 12 & 21,9 & 55 & 100,0 \\
\hline TOTAL & 398 & 81,0 & 93 & 19,0 & 55 & 100,0 \\
\hline
\end{tabular}

Em relação ao tipo de acidente encontramos um percentual significativo de $88,6 \%$ dos trabalhadores que sofreram acidente com instrumento perfurocortante. A grande objetos perfurocortantes registrando intensa exposição. $\mathrm{Na}$ exposição de contato por respingos em membranas emucosas uma mostra pequena, mas significativa de (11.4\%), conforme exposição aos fluidos biológicos ainda está no acidente com mostra a tabela 2:

Tabela 2 - Exposição a fluidos biológicos por acidentes com material perfurocortante e por respingos em membranas e mucosas. Curitiba, 2004.

\begin{tabular}{lcccccc}
\hline Mês & \multicolumn{2}{c}{ Perfurocortante } & \multicolumn{2}{c}{$\begin{array}{c}\text { Respingos em } \\
\text { mucosas/membranas }\end{array}$} & \multicolumn{2}{c}{ TOTAL } \\
\hline & $\mathrm{N}^{\circ}$ & $\%$ & $\mathrm{~N}^{\circ}$ & $\%$ & $\mathrm{~N}^{\circ}$ & $\%$ \\
\hline Abril & 22 & 10,4 & 2 & 0,9 & 24 & 11,4 \\
Maio & 8 & 3,8 & 4 & 1,9 & 12 & 5,7 \\
Junho & 22 & 10,4 & 3 & 1,4 & 25 & 11,8 \\
Julho & 24 & 11,4 & 0 & 0,0 & 24 & 11,4 \\
Agosto & 19 & 9,0 & 3 & 1,4 & 22 & 10,4 \\
Setembro & 21 & 10,0 & 4 & 1,9 & 25 & 11,8 \\
Outubro & 19 & 9,0 & 1 & 0,5 & 20 & 9,5 \\
Novembro & 24 & 11,4 & 3 & 1,4 & 27 & 12,8 \\
Dezembro & 28 & 13,3 & 4 & 1,9 & 32 & 15,2 \\
\hline TOTAL & 187 & 88,6 & 24 & 11,4 & 211 & 100,0 \\
\hline
\end{tabular}

Fonte: dados da pesquisa. 
Os resultados confirmam a alta exposição a agentes patógenos. A transmissão do HIV em profissionais de saúde foi associada, principalmente, aos acidentes com instrumentos pérfurocortantes ${ }^{(13)}$. A importância desses acidentes extrapola a ocorrência da simples lesão e adquire maior gravidade quando contaminados com sangue e secreções. Nessa situação, é reconhecida a possibilidade de transmissão de microrganismos patogênicos, capazes de gerar outros processos de desgaste, geral mente mais graves que o ferimento em si, dentre eles a Hepatite e a AIDS (2).

0 profissional de saúde deverá ser acompanhado pelo período de seis a 12 meses após exposição a fluidos biológicos por acidentes com material infectado pelo HIV e em acidentes com paciente-fonte desconhecido. Em exposições com paciente-fonte anti-HIV negativo, 0 acompanhamento do profissional acidentado deverá ser de no mínimo seis meses ${ }^{(30)}$. Embora existam estes dispositivos legais que buscam prevenir e preservar a saúde do trabalhador, garantindo seus direitos, na prática, estas medidas esbarram em sérias dificuldades, conforme encontramos na população estudada.

Em relação ao protocolo de acompanhamento preconizado pelo M inistério da Saúde e implantado no respectivo campo de estudo, apenas $175(35,6 \%)$ retornaram para a segunda consulta ambulatorial após 30 dias do acidente. Para a consulta previamente agendada após 90 dias do acidente apenas 103 (21\%). Os registros mostram que apenas 50 trabalhadores (10,2\%) fizeram 0 monitoramento e acompanhamento completo neste ambulatório, confirmando uma baixa adesão, conforme mostra a Tabela 3.

Tabela 3 - M onitoramento e acompanhamento completo dos trabal hadores após exposição com fluidos biológicos. Curitiba, 2004

Acompanhamento dos Trabalhadores Acidentados com Fluídos Biológicos

\begin{tabular}{lccccccccccc}
\hline \multicolumn{1}{c}{ Mês } & $\begin{array}{c}\text { Entrada no } \\
\text { PS }\end{array}$ & \multicolumn{2}{c}{$\begin{array}{c}\text { Retorno em 1 } \\
\text { mês }\end{array}$} & \multicolumn{2}{c}{$\begin{array}{c}\text { Retorno em } 90 \\
\text { dias }\end{array}$} & \multicolumn{2}{c}{$\begin{array}{c}\text { Retorno em } \\
180 \text { dias }\end{array}$} & \multicolumn{2}{c}{ Transferidos } & \multicolumn{2}{c}{$\begin{array}{c}\text { Abandono } \\
\text { com 30 dias }\end{array}$} \\
\hline \multirow{2}{*}{ Abril } & $\mathrm{N}^{\circ}$ & $\mathrm{N}^{\circ}$ & $\%$ & $\mathrm{~N}^{\circ}$ & $\%$ & $\mathrm{~N}^{\circ}$ & $\%$ & $\mathrm{~N}^{\circ}$ & $\%$ & $\mathrm{~N}^{\circ}$ & $\%$ \\
Maio & 69 & 18 & 26,1 & 9 & 13,0 & 8 & 11,6 & 1 & 1,4 & 0 & - \\
Junho & 41 & 8 & 19,5 & 6 & 14,6 & 4 & 9,8 & 3 & 7,3 & 0 & - \\
Julho & 52 & 21 & 40,4 & 15 & 28,8 & 10 & 19,2 & 6 & 11,5 & 0 & - \\
Agosto & 42 & 14 & 33,3 & 5 & 11,9 & 4 & 9,5 & 4 & 9,5 & 0 & - \\
Setembro & 47 & 19 & 27,9 & 12 & 17,6 & 7 & 10,3 & 3 & 4,4 & 2 & 2,9 \\
Outubro & 70 & 18 & 23,7 & 11 & 15,7 & 9 & 12,9 & 7 & 10,0 & 0 & - \\
Novembro & 47 & 25 & 53,2 & 19 & 40,4 & 3 & 6,4 & 6 & 12,8 & 0 & - \\
Dezembro & 55 & 29 & 52,7 & 15 & 27,3 & 0 & 0 & 5 & 9,1 & 0 & - \\
\hline TOTAL & 491 & 175 & 35,6 & 103 & 21,0 & 50 & 10,2 & 41 & 8,4 & 2 & 0,4 \\
\hline
\end{tabular}

Fonte: dados da pesquisa.

O acompanhamento sorológico anti-HIV (ELISA) deverá ser realizado no momento do acidente, sendo repetido após 6 e 12 semanas e em pelo menos 6 meses. A realização de teste anti-HIV deverá ser feita após aconsel hamento pré e pós-teste, devendo ser garantido ao profissional a confidencialidade dos resultados dos exames. A coleta para o teste anti-HIV, no momento do acidente, é importante, para posterior caracterização da infecção pelo HIV em decorrência do acidente profissional. Um profissional de saúde com teste anti-HIV reativo, no momento do acidente, deverá ser esclarecido que este resultado não se deve ao acidente e encaminhado para acompanhamento médico específico (30).

0 profissional deve ser acompanhado por um ano, especialmente, nas seguintes condições: sintomas de possível infecção aguda pelo HIV durante os primeiros seis meses de acompanhamento após o acidente, uma história clínica prévia sugerindo uma deficiência de resposta imune e a exposição ocupacional simultânea ao vírus da hepatite $C$. A inda, deverá ser orientado durante o período de 
acompanhamento para adotar medidas de possível prevenção de transmissão via sexual, utilizando preservativos, e através de sangue, e para evitar a doação de sangue/órgãos, gravidez e al eitamento materno ${ }^{(30)}$.

\section{CONSIDERAÇÕES FINAIS}

Este estudo teve a intenção de subsidiar a reflexão dos administradores e dos trabal hadores de saúde para 0 desenvolvimento de estratégias que possibilitem minimizar a exposição aos riscos a que estes trabalhadores estão expostos e maximizar os recursos institucionais para a real ização do monitoramento completo quando expostos a fluidos biológicos.

A Igumas estratégias na instituição já foram implantadas para o completo acompanhamento e monitoramento destes trabalhadores expostos a fluidos biológicos, mas afirmamos que na prática, estas estratégias não dependem apenas de um serviço de saúde. Dependem, também, de outras medidas que possibilitem a conscientização destes trabalhadores na compreensão de seu processo de saúde, sejam por parte dos próprios trabal hadores, bem como nos seus locais de trabal ho.

ABSTRACT: Epidemiological research, cohort study with a quantitative approach, which objectified: characterize the health workers' profile injured with biological fluids and to analyze their compliance to the established protocol by returning to the outpatient clinic for consultation after exposure. The study field was The Worker's Outpatient Clinic at The Worker's Hospital from Federal University of Paraná located in the city of C uritiba, Paraná State, B razil. Four hundred and ninety-one (491) records were analyzed from A pril to December/ 2004. Data were collected through follow-up records of accidents with biological material and through the emergency reports from the Emergency ward of this institution. Data analysis evidenced that the most subjects were female $(80.4 \%)$, average age between 21 to 25 years $(25.8 \%)$. The nursing practitioners were the most incident category $(46 \%)$, followed by janitors in health units $(12 \%)$. From those 491 records of the Emergency Ward for working place exposure to biological fluids, only 175 $(35.6 \%)$ of the workers returned to their second appointment, after 30 days of the accident; 103 (21\%) for the previously- fixed appointment after 90 days, and only $50(10.2 \%)$ workers completed the follow -up and monitoring in the clinic. The accidents mostly happened due to contact with sharp- pointed instruments (88.6\%). J ust a small sample (11.4\%) had eye membrane spills of biological fluids. We believe this can be a thought-provoking study for health managers and professionals to work out strategies to minimize health workers' risk exposure as well as to maximize institutional resources in order to fully monitor them when exposed to biological fluids.
KEY WORDS: Work accidents; Occupational monitoring.

RESUMEN: Esta es una investigación epidemiológica, un etudio con abordaje cuantitativo, con los siguientes objetivos: caracterizar el perfil de los trabajadores víctimas de accidentes con fluidos biológicos y analizar la adhesión en el retorno al ambulatorio para consulta después de la exposición ocupacional. La institución que servió como campo de estudio fue el A mbulatorio del Trabajador que pertenece al Hospital do Trabalhador da Universidade Federal do Paraná. Fueron analizados prontuarios en el periodo de abril a diciembre de 2004. Los datos fueron recogidos por medio de la "Ficha de A compañamiento de Accidente con Material Biológico" y del "Boletín de Emergencia en el Hospital de U rgencias" de esta institución. Los datos demuestran que la mayoría de los individuos pertenece al sexo femenino $(80,4 \%)$, con media de edad de 21 a 25 años (25,8\%). La categoría auxiliar de enfermería fue la que más tuvo contacto con fluidos biológicos (46\%), los funcionarios que ejecutan actividad de limpieza en unidades de sal ud también fueron víctimas de accidente en una muestra significativa (12\%). De los prontuarios registrados que recurrieron al Servicio de Emergencia por exposición ocupacional, solamente $175(35,6 \%)$ volvieron a la segunda consulta ambulatorial después de 30 días del accidente. Para las consultas marcadas para después de 90 días del accidente, sólo $103(21 \%)$ ). L os registros mostraron que solamente 50 trabajadores $(10,2 \%)$ hicieron la monitorización y acompañamiento completo en el ambulatorio. En relación al tipo de accidente, el porcentual encontrado fue significativo: $88,6 \%$ de los trabajadores que fueron víctimas de accidente con instrumento perforante cortante. Sól o una pequeña muestra de $11,4 \%$ tuvo contacto con los fluidos biológicos por medio de salpicaduras en mucosas oculares. Es posible que este estudio pueda subsidiar la reflexión de los administradores y trabajadores de salud para el desarrollo de estrategias que posibiliten minimizar la exposición a los riesgos a que eses trabajadores están expuestos y maximizar los recursos institucionales para la realización de la monitorización completa cuando expuestos a los fluidos biológicos.

PALABRAS CLAVES: Accidentes de Trabajo; M onitorización Ocupacional.

\section{REFERÊNCIAS}

1. Secretaria do Estado do Paraná. Instituto de Saúde do Paraná. Saúde do Trabalhador. [on line]. Disponível na Internet: http:// www.saude.pr.gov.br/ (02/06/04)

2. Silva VEF. O desgaste do trabalhador de enfermagem: estudo da relação trabalho de enfermagem e saúde do trabalhador. [tese] São Paulo (SP): Escola de enfermagem, U niversidade de São Paulo; 1996.

3. M arziale M HP. Estudo da fadiga mental de enfermeiras atuantes em

Cogitare Enferm 2005 mai/ago; 10(2):47-53 
instituição hospitalar com esquema de trabalho em turnos alternantes. [dissertação]. Ribeirão Preto (SP): Faculdade de Filosofia de Ciências e L etras de Ribeirão Preto, U niversidade de São Paulo; 1990.

4. Silva VEF. Estudo sobre acidentes de trabal ho ocorridos com trabal hadores de enfermagem de um hospital de ensino. [dissertação]. São PauIo: Escola de Enfermagem, Universidade de São Paulo; 1988.

5. B arbosa A. Riscos ocupacionais em hospitais: um desafio aos profissionais da área de saúde ocupacional. [dissertação]. Florianópolis (SC): Universidade Federal de Santa Catarina; 1989.

6. A lexandre NM C. Contribuição ao estudo das cérvicodorsolombal gias em profissionais de enfermagem. [tese]. Ribeirão Preto (SP): Escola de Enfermagem de Ribeirão Preto, U niversidade de São Paulo; 1993.

7. Marziale M HP. Condições ergonômicas da situação de trabalho, do pessoal da enfermagem, em uma unidade de internação hospitalar. [tese]. Ribeirão Preto (SP): Escola de Enfermagem de Ribeirão Preto, Universidade de São Paulo; 1995

8. Souza M. Conhecimento e aplicação das precauções universais pelos componentes da equipe de enfermagem de um hospital governamental. [dissertação]. São Paulo (SP): Escola Paulista de M edicina, Universidade Federal de São Paulo; 1995.

9. Silva A. Trabal hador de enfermagem na U nidade de $C$ entro de $M$ aterial e os acidentes de trabalho. [tese]. São Paulo (SP): Escola de Enfermagem, Universidade de São Paulo; 1996.

10. Benatti MCC. A cidente do trabalho em um hospital universitário: um estudo sobre a ocorrência e os fatores de risco entre os trabal hadores de Enfermagem . [tese]. São Paulo (SP): Escola de Enfermagem de São Paulo, Universidade de São Paulo; 1997.

11. B revidelli M M . Exposição ocupacional ao vírus da AIDS e da hepatite $B$ : análise da influência das crenças em saúde sobre a prática de reencapar agulhas. [dissertação]. São Paulo (SP): Escola de Enfermagem , U niversidade de São Paulo; 1999.

12. Faria LS, Silva VEF. Os riscos fisiológicos e psíquicos identificados entre os trabalhadores de enfermagem em um centro de reabilitação. In: Programa do $10^{\circ}$ Seminário de Pesq em Enf; 1999; Gramado. Gramado: A BEn-Sec RS; 1999.

13. Sarquis LMM. Acidentes de trabalho com instrumentos perfurocortantes: ocorrência entre os trabalhadores de enfermagem. [dissertação]. São Paulo (SP): Escola de Enfermagem, Universidade de São Paulo; 1999.

14. Sarquis LM , Felli VEA. O uso do equipamento de proteção individual entre os trabal hadores de enfermagem acidentados com instrumentos perfurocortantes. Rev Bras Enferm 2000; 53(4):564-73.

15. Sarquis LM, Felli VEA. A cidente de trabalho com instrumento perfurocortantes entre os trabalhadores de enfermagem. Rev Escola Enferm USP 2002; 36(3):222-30.

16. Rocha AM . A saúde do trabalhador de enfermagem sob a ótica da gerência: obstáculos e possibilidades. [tese]. São Paulo (SP): Escola de Enfermagem da Universidade de São Paulo; 2003.

17. Canini SRMS, Gir E, Hayashida M, Machado AA. A cidentes perfurocortantes entre os trabal hadores de enfermagem de um hospital universitário do interior paulista. Rev Latino-am Enferm 2002; 10(2):172-8.

18. Sêcco IA O, Robazzi M LCC, Gutierres PR, M atsuo Tiemi. A cidentes de trabal ho e riscos ocupacionais no dia-a-dia do trabalhador hospitalar: um desafio para a saúde do trabalhador, Espaço para Saúde 2002; 4(1).

19. Costa TF. Exposição dos trabal hadores de enfermagem às substância químicas: estudo em um hospital público universitário. [dissertação]. São Paulo (SP): Escola de Enfermagem da Universidade de São Pau10; 2002.

20. Rocha FLS, M arziale MHP, Robazzi M LCC. Perigos potenciais a que estão expostos os trabal hadores de enfermagem na manipulação de quimioterápicos antineoplásicos: conhece-los para previní-los. R ev Latino-am Enferm 2004; 12(3):511-7.

21. Silveira CA, Zapparoli AS, Robazzi M LCC, M arziale M HP. Produção do conhecimento sobre riscos do trabal ho de enfermagem e sua divulgação em base de dados disponibilizadas pela internet. In: Resumo do Programa Científico Ribeirão Preto: EERP; 2004

22. Ribeiro EJC. Estudo de acidentes de trabalho com trabalhadores de enfermagem de um hospital-escola do Distrito Federal. [dissertação] Brasília (DF): Universidade de Brasília;2004.

23. Nishide VM, B enatti M CC. Riscos Ocupacionais entre trabal hadores de enfermagem de uma Unidade de Terapia Intensiva. Rev Esc Enferm USP 2004; 38 (4): 406-14.

24. Zurita IEO. Segurança do trabal ho em ambientes hospitalares. Rev CIPA 1993; 4:20-30.

25. Zocchetti C, Ferrario M . M edicazioni e franchigie: aspetti pouco noti del fenomeno infortunístico. M ed Lav 1994; 85(3):205-18.

26. Rowe MP, Giuffre M. Evaluating needlestick injuries in nursing personnel. A A OHN 1991; 39(11):503-7.

27. Centers For Disease Control A nd Prevention. Guideline for infection control in health care personal. Infect. Control in Hospital Epidemiol. 2001; 19(6):445

28. Centers For Disease Control A nd Prevention. CDC.gov - Occupational exposures needlestick injure. Available: http://www.cdc.gov/ hivserch.htm A ccess at: jan, 2006.

29. Polit $D$, Hungler BP. Pesquisa em Enfermagem: princípios e métodos. Porto A legre: A rtes M édicas; 1995.

30. M inistério da Saúde (BR). Secretaria de Políticas de Saúde. Coordenação Nacional de DST e AIDS. Recomendações para terapia antiretroviral em adultos eadolescentes infectado pelo HIV. B rasília; 2002.

ENDERÇO DOSAUTORES: Av. Iguaçu, 2666 - ap. 1201 Curitiba/PR 80240-030 m.sarquis@brturbo.com 